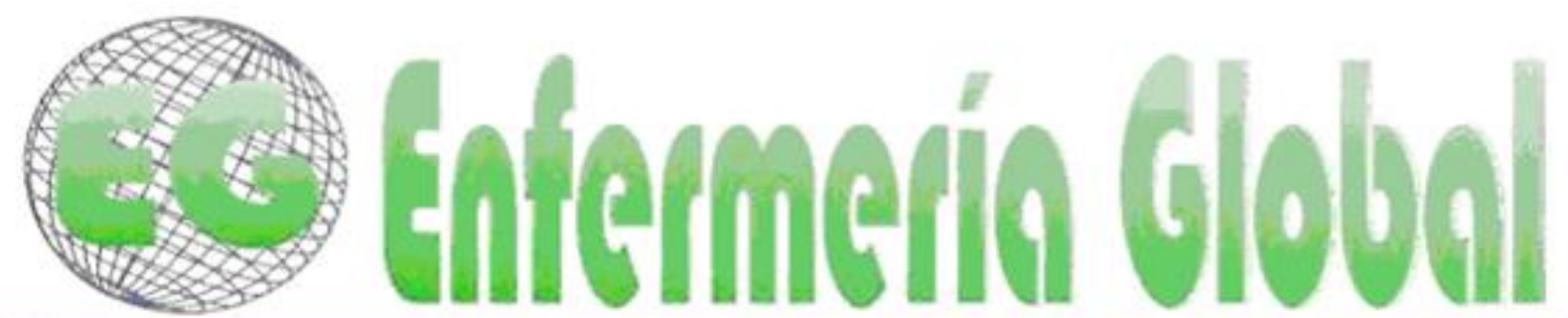

\title{
Prevalencia y factores asociados a osteoporosis en pacientes de Santa Marta (Colombia)
}

Prevalence and factors associated with osteoporosis in patients of Santa Marta (Colombia)

\section{* González-Ruiz, Gisela Esther **Álvarez-Miño, Lídice ***Borré-Ortiz, Yeis}
Miguel ${ }^{* * *}$ Rivas Oduber, Elimiled ${ }^{* * * *}$ Serrano Angarita, Nini Sorell ****Tavera Galeano, Nivalisay

\begin{abstract}
*Enfermera. Magister en Ciencias Básicas Biomédicas. Docente Facultad de Enfermería Universidad Cooperativa de Colombia, sede Santa Marta. E-mail: gisela.1060@gmail.com **Magister en Salud Pública, Docente Facultad de Enfermería Universidad del Magdalena, Santa Marta. ***Magister en Enfermería. Docente Facultad de Enfermera Universidad Cooperativa de Colombia, sede Santa Marta ****Enfermera. Facultad de Enfermería Universidad Cooperativa de Colombia, sede Santa Marta. Colombia.
\end{abstract}

\section{Palabras clave: Osteoporosis; factor de riesgo; densitometría; hueso (Fuente: DeCS Bireme) Keywords: Osteoporosis; risk factor; densitometry; bone}

\section{RESUMEN}

Objetivo: Identificar la prevalencia y los factores asociados a osteoporosis en un grupo de pacientes Samarios.

Materiales y Método: Estudio descriptivo, retrospectivo y correlacional, realizado en una población de 406 pacientes de un total de 1486 consultantes.

Resultados: La prevalencia encontrada fue de 27,32; los factores asociados fueron: edad mayor de 50 años $(p=0,3405)$; género femenino $(p=0,3405)$, no realización de ejercicio físico $(p=0,011)$; paridad $(p=$ 0,0001 ). Se halló asociación estadística entre la osteoporosis y las enfermedades del ojo y sus anexos $(p=0,0415)$, según la clasificación del Consejo Internacional de Enfermedades.

Conclusiones: La prevalencia de osteoporosis fue de 27,32\%. La edad, el sedentarismo, la multiparidad, las enfermedades de los ojos y sus anexos, los antecedentes de cirugías ginecobstétricas y de vesícula fueron factores de riesgo estadísticamente significativos para el desarrollo de la osteoporosis. 


\section{ABSTRACT}

Objective: To identify the prevalence and factors associated with osteoporosis in a group of patients of Santa Marta.

Materials and Method: Retrospective descriptive and correlational study in a population of 406 patients from a total of 1486 consultants.

Results: The prevalence found was 27.32; associated factors were: age higher than 50 years $(p=$ $0.3405)$, female gender $(p=0.3405)$, no physical exercise $(p=0.011)$, parity $(p=0,0001)$. Statistical association was found between osteoporosis and diseases of the eye and adnexa $(p=0.0415)$, by classification International Council Disease.

Conclusions: The prevalence of osteoporosis was $27.32 \%$. Age, sedentary lifestyle, multiparity, diseases of the eye and its annexes, the background to ginecobst and gallbladder surgeries were statistically significant risk factors for developing osteoporosis.

\section{INTRODUCCIÓN}

La osteoporosis es una enfermedad metabólica sistémica, caracterizada por disminución de la masa ósea y deterioro de la microarquitectura del hueso que produce aumento de su fragilidad (1) y afecta la calidad y la cantidad del órgano. En Estados Unidos, esta enfermedad ataca aproximadamente a seis millones de mujeres y dos millones de hombres; lo que representa un gasto anual superior a 10 mil millones de dólares. Adicionalmente, es causante del $20 \%$ de fracturas en mujeres postmenopáusicas de dicho país y de 7,8 millones a nivel mundial ${ }^{(2)}$.

El riesgo de sufrir una fractura por esta causa, a partir de los 50 años, es de $40 \%$ en mujeres y $13 \%$ en hombres. La edad es un factor representativo en ambos sexos ${ }^{(3)}$. En España, el $12,73 \%$ de la población femenina tienen osteoporosis, lo que representa alrededor de 1.974 .400 casos ${ }^{(4)}$. En Colombia, específicamente Bogotá, reporta una prevalencia que oscila entre el $32,3 \%$ y el $57 \%{ }^{(5)}$. Dicha prevalencia aumenta con la edad, desde el $15 \%$ para las edades comprendidas entre 50 y 59 años, hasta una prevalencia mayor del $80 \%$ para las mujeres con una edad superior a los 80 años ${ }^{(6)}$.

El proceso de resorción ósea se acelera con la menopausia y alcanza una disminución entre un $2 \%$ a $5 \%$ anual, dentro de los primeros 5 a 10 años ulteriores a la amenorrea mantenida ${ }^{(7)}$. El calcio se conserva predominantemente en el esqueleto (1-2 Kg). Sin embargo, normalmente una persona pierde $250 \mathrm{mg}$ diarios de calcio a través de la piel, el intestino y los riñones ${ }^{(8)}$. El cuello del fémur es el sitio que predice mejor el riesgo de fractura de cadera y de otros sitios esqueléticos ${ }^{(2)}$.

La densidad mineral ósea posee 4 categorías: normal (valor DMO normal superior de -1 desviación estándar de la referencia para adultos); osteopenia o baja masa ósea (Valor de DMO de -1 a -2.5 desviaciones estándar); osteoporosis (valor de DMO menor de -2.5 desviaciones estándar) y osteoporosis establecida o severa (valor de DMO en combinación con una o más fracturas) (9).

El tratamiento consiste en llevar una dieta adecuada a base de calcio, ejercicio físico, eliminación de tóxicos con efectos sobre el hueso y suministro de vitamina D3 ${ }^{(10)}$; además de terapia sustitutiva hormonal ${ }^{(11)}$. La prevalencia puede ser mayor en mujeres posmenopáusicas de piel blanca (12), edad mayor de 50 años, postmenopáusicas, peso menor de 50 kilos, antecedente de fractura y menos de 50 kilos de peso, como factor de riesgo asociado ${ }^{(4,13,14)}$. 
Un estudio reportó como factores asociados la edad mayor de 18 años, el hipogonadismo y la ingesta cálcica inferior a $500 \mathrm{mg} /$ día. Los factores menos representativos fueron el tabaquismo, el índice de masa corporal inferior a $25 \mathrm{Kg} / \mathrm{m}^{2}$, antecedente de fracturas, menopausia precoz, tratamiento con glucocorticoides por más de 6 meses, enfermedades como la diabetes tipo II y artritis reumatoide (15). Mientras que en otro grupo de pacientes estudiados, la mayor prevalencia la tuvieron las mujeres mayores de 65 años, el $37,8 \%$ con osteoporosis primaria y el $46 \%$ secundaria ${ }^{(16)}$. En otros estudios no se mostró relación significativa entre el cigarrillo, el consumo de corticoides y la artritis reumatoide, pero predominó la edad mayor de 50 a 70 años ${ }^{(17)}$, con prevalencia de osteoporosis de $15 \%{ }^{(18)}$.

Balderramo et al. ${ }^{(19)}$ hallaron que el $40 \%$ de las mujeres estudiadas eran fumadoras, el $47 \%$ recibía reemplazo hormonal durante el periodo posmenopausia, y el $31,6 \%$ estaban histerectomizadas; mientras que Padierna reporta como factores asociados la edad, los antecedentes familiares de osteoporosis, menopausia mayor de 10 años, poco ejercicio físico, peso inferior a $56 \mathrm{~kg}$, ingestión de café y tabaquismo ${ }^{(20)}$.

Rosen, en su estudio con 707 mujeres, evidencia una prevalencia de osteopenia y osteoporosis de $32,3 \%$ y $51,8 \%$ respectivamente. Además, observó que el $40,3 \%$ tenía edades entre 50 y 59 años, con antecedentes quirúrgicos generales y ginecológicos $(78,6 \%)$, con antecedentes osteoarticulares $(62,1 \%)$ y endocrinos $(22,3 \%)^{(6)}$. Otro estudio efectuado en 5.924 pacientes, mostró un total de $17,9 \%$ pacientes con osteoporosis $\left(79,8 \%\right.$ mujeres y $20,1 \%$ hombres) ${ }^{(21)}$. Por otra parte, Bocanegra et al. ${ }^{(22)}$ hallaron una prevalencia de osteoporosis del $20,6 \%$, en un grupo de pacientes de 37 - 47 años. Ponce et al ${ }^{(23)}$ encontraron que el $57,9 \%$ de personas con osteoporosis posee menor índice de masa corporal, y que además, la multiparidad hace mayor la densidad de masa ósea, mientras que con la nuliparidad es menor. Por su parte, Díaz et al ${ }^{(24)}$ observaron una prevalencia de osteoporosis de $29,14 \%$.

A pesar de los datos registrados, la ciudad de Santa Marta no cuenta con estudios en los que se hayan analizado estadísticas y factores asociados a la osteoporosis. Es importante evidenciar estos datos, los cuales servirán como referencia o antecedente para futuras investigaciones; permitiendo además, comparar la prevalencia de la alteración con referentes Nacionales e Internacionales.

En consecuencia, este estudio buscó identificar la prevalencia de osteoporosis en le ciudad de Santa Marta, además de contextualizar los factores de riesgo asociados a su incidencia.

\section{MATERIALES Y MÉTODO}

Estudio descriptivo, retrospectivo y correlacional, efectuado en una muestra censal de 406 pacientes de un total de 1.486. Los criterios de inclusión establecidos fueron: pacientes con registro de densitometría ósea, información de registro completo, diagnóstico de osteoporosis y haber sido atendido en la institución objeto de estudio. Se excluyeron aquellos casos con diagnóstico de osteopenia.

La recolección de los datos se hizo mediante la revisión de la base de datos de la entidad, cuya información es validada e introducida por los médicos que allí laboran. Dicha base de datos contiene información relacionada con variables 
sociodemográficas y de estilos de vida (consumo de cigarrillo, alcohol, café y ejercicio), antecedentes personales (embarazo y menstruación), antecedentes patológicos (enfermedad, cirugías practicadas, medicamentos), diagnóstico de osteoporosis, según la definición de la Organización Mundial de la Salud.

Para el análisis univariado de la información se utilizó el programa Microsoft Excel®, y para el análisis bivariado el programa Epi-Info versión 3.5.1 del 2008. Los estadísticos descriptivos utilizados fueron la frecuencia absoluta y relativa, y los estadísticos de asociación fueron: la prueba de $\mathrm{Chi}^{2}$, con un valor " $\mathrm{p}$ " de significancia estadística $(p<0,05)$, un grado de libertad $y$, la OR (odds ratio). Teniendo en cuenta la siguiente interpretación: valor nulo $(O R=1)$, factor protector $(O R<1)$ y factor de riesgo $(O R>1)$.

Como medida ética se garantizó la confidencialidad en el manejo de la información suministrada por la entidad, respetando los criterios establecidos en la Resolución 8430 de 1993 (25) y la declaración de Helsinki (26).

\section{RESULTADOS}

La prevalencia de osteoporosis encontrada fue de $27,32 \%$, con predominio en el género femenino (95\%).

Tabla I. Distribución de la información según variables: Diagnóstico, Género y Edad

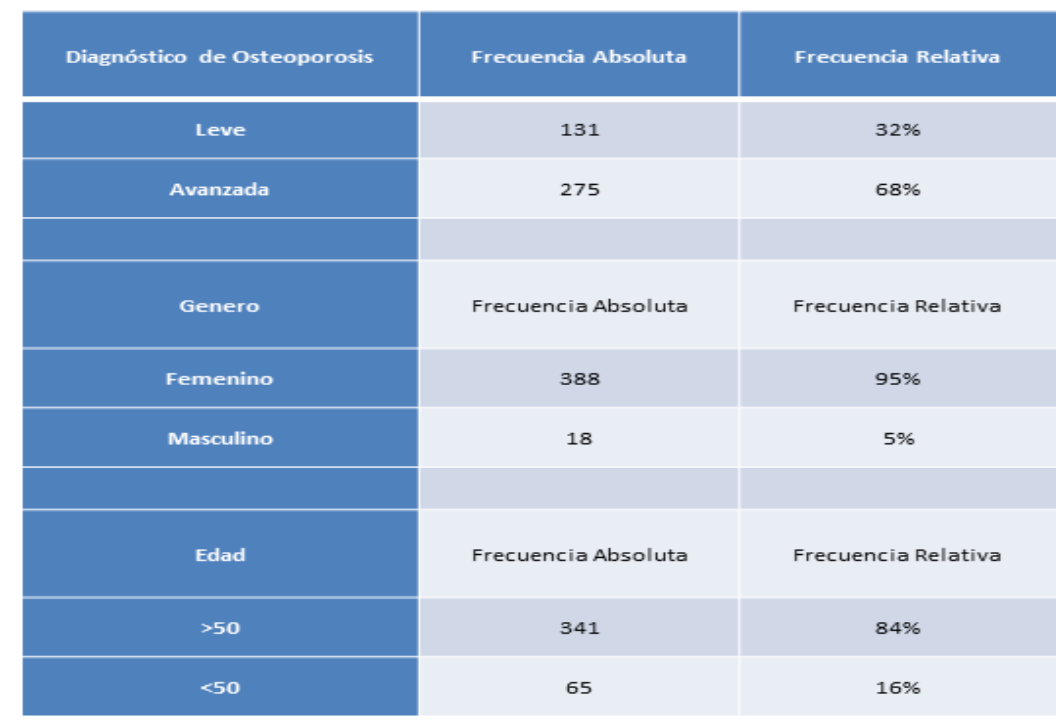

Fuente: Base de datos de la Institución. 2012

El $32 \%$ de los pacientes fue diagnosticado con osteoporosis leve y el $68 \%$ con osteoporosis avanzada. El 16\% era menor de 50 años y el $84 \%$ mayor de 50 años. De éstos, el 34\% tenía edades mayores a los 65 años.

El análisis estadístico bivariado mostró que la edad $(p=0,6737)$ y el sexo $(p=0,3405)$ son variables asociadas a la presencia de osteoporosis $(\mathrm{OR}=1,610$ y 1,232 ) respectivamente. 
Gráfico 1. Asociación entre hábitos de consumo de alcohol, tabaco y café

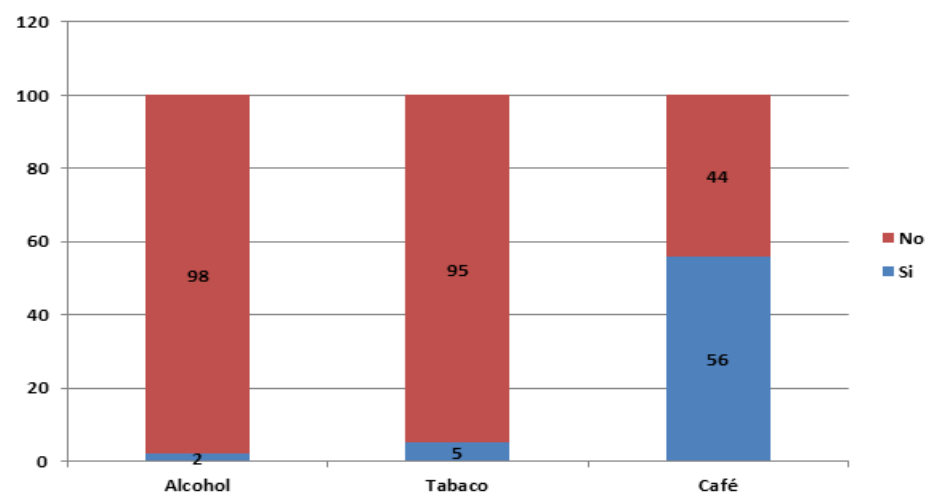

Fuente: Base de datos de la Institución. 2012

Al momento del estudio el $56 \%$ consumía café, el $5 \%$ tabaco y el $2 \%$ alcohol. El consumo de cigarrillo, alcohol y cafeína no evidenció asociación estadística significativa ( $p=0,2247 ; p=0,9681 ; p=0,5190)$ respectivamente. Sin embargo, la prueba de OR: 1,6981 indica que sí son factores asociados a la enfermedad. A la población fumadora se le atribuyó el 1,7 de probabilidad de padecer la enfermedad con respecto a la población que no fumaba.

Grafico 2. Asociación entre la práctica de ejercicio y la osteoporosis

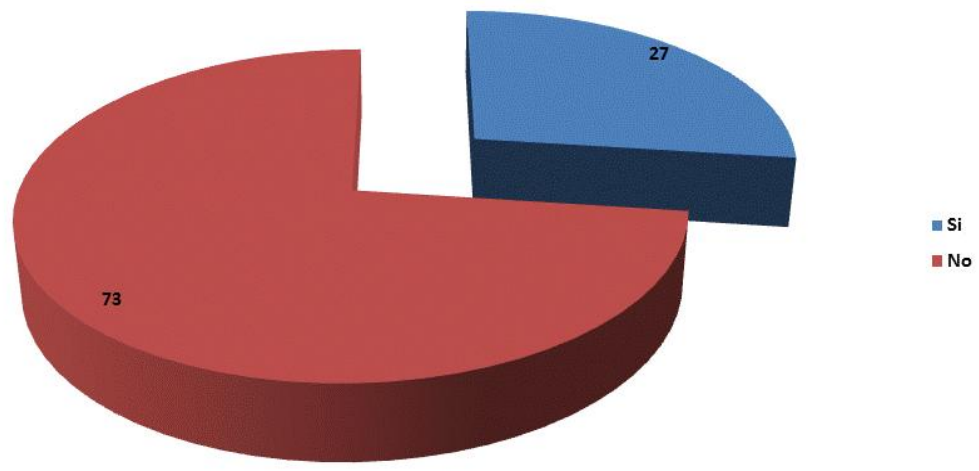

Fuente: Base de datos de la Institución. 2012

La información recolectada mostró que ninguno de los participantes diagnosticados con osteoporosis avanzada y leve practicaba algún tipo de ejercicio físico $(p=0,011)$, pero si se halló que existe dependencia entre la variable ejercicio con la variable osteoporosis $\left(\mathrm{Chi}^{2}=6,4355 ; \mathrm{OR}=1,7625\right)$, convirtiéndose en un factor de riesgo asociado a osteoporosis y aumentando en un 1,8 la probabilidad de sufrir osteoporosis.

El $11 \%$ no registró historia de embarazos, el $63 \%$ entre 1 y 5 , el $22 \%$ entre 6 y 10 , el $3 \%$ entre 11 y 15 y el $1 \%$ más de 15 . Un $99 \%$ se encontraba en etapa postmenopáusica y un $1 \%$ en ciclo menstrual. Al relacionar la variable de 
menstruación con la presentación de osteoporosis no se encontró significancia estadística $(p=0,4754)$.

Los antecedentes patológicos, según el Código Internacional de Enfermedades (CIE), reportó $26 \%$ enfermedades cardiovasculares, $20 \%$ dolor no especificado, $18 \%$ alteraciones del sistema osteomuscular y tejido conectivo, y $10 \%$ enfermedades endocrinas, nutricionales y metabólicas.

Al $51 \%$ le fue practicada algún tipo de cirugía gineco-obstétrica y a 3 hombres se les había practicado cirugía de próstata. La variable (enfermedades del ojo y sus anexos) con código H00 - H59, mostró significancia estadística $\left(p=0,0415\right.$; $\left.\mathrm{Chi}^{2}=4,1535\right)$, con un OR indeterminado. Es decir, que esta variable se descarta como factor de riesgo asociado a la osteoporosis. No obstante, llama la atención que las enfermedades (Trastornos mentales y del comportamiento) con código F00 - F99, a pesar de no tener significancia estadística evidente, se convirtieron en un factor de riesgo para la población estudiada según valores de osteoporosis $(9,81)$. Relación que podría atribuirse a la dinámica farmacológica que ejercen los antipsicóticos disminuyendo los estrógenos y la testosterona.

Los antecedentes de cirugías gineco-obstétricas mostraron significancia estadística con la osteoporosis $(p=0,0042)$ y una dependencia $\left(\mathrm{Chi}^{2}=8,1926\right)$ que lo constituye como factor agregado a la presentación de osteoporosis, pero no como factor de riesgo $(\mathrm{OR}=0,559)$. Las cirugías de vesícula fueron estadísticamente significativa $(\mathrm{p}=$ $0,0302)$, pero no hubo relación interdependiente con el diagnostico de osteoporosis $\left(\mathrm{Chi}^{2}=4,6965\right)$. Sin embargo, posiblemente pudo convertirse en un factor de riesgo para la población estudiada ( $\mathrm{OR}=2,698)$.

Finalmente, las cirugías practicadas por trauma o fractura resultaron ser estadísticamente no significativa con respecto a la presencia de osteoporosis $\left(p=0,4363 ; \mathrm{Chi}^{2}=0,6060\right)$. Sin embargo, se pudo asociar como factor de riesgo en los pacientes que cursaron con la enfermedad $(\mathrm{OR}=1,353)$.

El consumo de medicamentos según valores "p", $\mathrm{Chi}^{2}$ y OR no fue concluyente, ya que un porcentaje significativo de los participantes no los usaba al momento del estudio.

\section{DISCUSIÓN}

La prevalencia de osteoporosis en el grupo de pacientes estudiados es de $27,32 \%$ y los factores asociados fueron: edad mayor de 50 años, sexo femenino, la no práctica de ejercicio, número de embarazos, enfermedad de ojos y anexos, cirugías ginecoobstétricas y de vesícula. Hallazgos confirmados mediante las pruebas estadísticas utilizadas. La edad de aparición se explica por los procesos fisiológicos que se dan en los seres humanos en relación a la disminución de la densidad del hueso y el aumento de la prevalencia en género femenino puede estar relacionada con los procesos hormonales que aparecen después de la menopausia.

La prevalencia encontrada en este estudio (27,23\%) es alta, si se compara con la presentada por pacientes españoles y mexicanos en estudios previos (12,73\%$20,6 \%)^{(5,17,22,24)}$, pero aun así, se encuentra por debajo de la reportada en estudios anteriores realizados en Bogotá $(57 \%)^{(7)}$, Chile $(57,9 \%)$, Cuba $(34 \%)$ y Mayorca 
$(44,2 \%)^{(4,12,24)}$. La variación de la prevalencia puede estar asociada con factores socio-culturales.

La osteoporosis leve y avanzada se presenta en mujeres mayores de 50 años, pero en ninguno de los casos se presentó significancia estadística, sino que según valores de OR fueron factores asociados. Dato concordante con lo hallado en otros estudios (22, 27-29). Los hábitos de consumo de alcohol y cafeína no mostraron significancia estadística, pero la ausencia de ejercicio si índica un aumento de 1,8 en la probabilidad de padecer la patología; mientras que el consumo de cigarrillo aumenta la probabilidad en 1,7.

La literatura revisada sobre el tema muestra que el uso moderado de alcohol etílico puede tener efectos benéficos sobre la densidad mineral ósea, independiente del sexo y la edad. Lo cual indica que no es un factor de riesgo osteoporótico (30), mientras que el consumo de tabaco puede ser factor causal ${ }^{(31,32)}$.

El ejercicio representa un factor protector si se desarrolla por más de 30 minutos durante 3 veces al día ${ }^{(14)}$, puesto que evita los procesos osteogénicos, proporciona un mayor pico de masa ósea en edad juvenil y previene las pérdidas de masa mineral en edades avanzadas; aspecto que lo relaciona como factor protector con el mantenimiento de la masa ósea ${ }^{(33)}$.

El ciclo menstrual no mostró significancia estadística. Sin embargo, la menopausia si se constituye un factor asociado a la osteoporosis; dato evidenciado por otros autores $\left.{ }^{(34,31}\right)$. Mientras que el número de embarazos presentó una relación estadísticamente significativa, con un alto grado de dependencia.

En un estudio de factores de riesgo modificables o no, relacionados con la densidad mineral ósea en mujeres de edad mediana con respecto al embarazo, evidenció que el $91,5 \%$ del total de la muestra tuvo embarazos a término. Este antecedente no demostró diferencia entre los grupos estudiados, pero en el presente estudio, el hecho de tener antecedentes de más de cuatro embarazos, se constituyó en un factor protector para la osteoporosis. Hallazgo que puede asociarse con la adaptabilidad del organismo de la mujer, ajustando la homeostasis del calcio para favorecer un balance positivo ${ }^{(8)}$, como resultados de adaptaciones metabólicas que aseguran el adecuado suministro de calcio al feto.

Es verdad que la masa ósea no disminuye durante el embarazo, pero puede darse con la lactancia materna sostenida por más de 6 meses ${ }^{(35)}$.

Uno de los antecedentes patológicos estadísticamente significativos fueron las enfermedades del ojo y sus anexos. No obstante, llama la atención que, aunque esta fue una variable con significancia estadística sin dependencia con el diagnóstico de osteoporosis, no se considera motivo causal para padecer la osteoporosis. Al respecto, no existen datos científicos que expliquen la relación entre este grupo de enfermedades y la osteoporosis.

Por otra parte, los trastornos mentales y del comportamiento, a pesar de que no tuvieron significancia estadística, se convirtieron en un factor de riesgo para la población estudiada; aspecto que se puede explicar por el uso de medicamentos anticonvulsivantes (tipos fenitoína). Sin embargo, estos datos no fueron corroborados en este estudio. 
De igual forma, los resultados relacionados con los antecedentes de cirugías ginecoobstétricas y vesícula mostraron significancia estadística. Las primeras podrían estar asociadas con las modificaciones hormonales derivadas de este tipo de cirugías. Con respecto a las cirugías por fracturas y traumas, no se presentó significancia estadística, pero si fueron un factor de riesgo para padecer osteoporosis. La literatura revisada mostró hallazgos de afectación articular en la enfermedad inflamatoria intestinal por manifestaciones extradigestivas, especialmente en el esqueleto, piel y ojos, las cuales se denominan manifestaciones articulares de la Ell ${ }^{(36)}$.Pero, es necesario seguir investigando dicha relación para confirmar si se trata de una enfermedad relacionada con el tipo de alimentación consumida, y que podría estar afectando ambos sistemas.

El uso de medicamentos no fue concluyente ya que un porcentaje alto de los participantes no recibía tratamiento.

Es importante hacer seguimiento oportuno a los pacientes que se les diagnostique osteoporosis y establecer un plan de prevención, especialmente en aquellos casos con osteopenia y osteoporosis leve, que además de la administración del tratamiento, retrase la evolución progresiva de la enfermedad. Asimismo, es importante establecer programas de prevención y control de la osteoporosis, como parte de las actividades obligatorias emanadas desde el Ministerio de Salud y Protección Social. Actividades con las que se registre y haga seguimiento a todos los pacientes que tengan factores de riesgo de padecer la enfermedad; así como modificar hábitos asociados y disminuir el riesgo de padecer la patología.

Es necesario incluir el ejercicio como hábito y estilo de vida saludable, coadyuvando a la búsqueda activa de casos. Estos resultados permitirían divulgar el comportamiento específico de la osteoporosis en Santa Marta (Colombia) y comparar la prevalencia con la presentada a nivel regional, nacional y mundial.

Por otra parte, es indispensable el desarrollo de nuevas investigaciones con poblaciones de ambos géneros, en las que se utilicen métodos estadísticos aleatorios que permitan profundizar en los resultados, ya que al elegir técnicas de muestreo intencional, las inferencias estadísticas son menos posibles. Además, la información debe obtenerse de fuentes primarias para superar las limitaciones del presente estudio.

\section{CONCLUSIONES}

La prevalencia de osteoporosis en población adulta de Santa Marta, Colombia, fue mayor a la encontrada por otros estudios en diferentes países del Mundo. Sin embargo, fue más baja que la hallada en otras regiones colombianas. La edad, el sedentarismo, la multiparidad, las enfermedades de los ojos y sus anexos, los antecedentes de cirugías ginecobstétricas y de vesícula fueron factores de riesgo estadísticamente significativos para el desarrollo de la osteoporosis.

\section{REFERENCIAS}

1. Ministerio de Sanidad, Política Social e Igualdad. Guías de práctica clínica sobre osteoporosis y prevención de fracturas por fragilidad. 2009. consultado el 
http://www.guiasalud.es/GPC/GPC 476 Osteoporosis AIAQS compl.pdf

2. Forero E. Osteoporosis secundaria y osteoporosis inducida por glucocorticoides. Salud Uninorte. 2006; 22(2): 121-34.

3. Guzmán J, Flores R, Gómez J, Suberanes E, Escarela M, d'Hyver de las Deses C. Osteoporosis, conceptos básicos para la práctica diaria. Rev. Especial Med. Quirúrg. 2009; 14(3): 128-40.

4. Más A, Cabanes T, Mari B. Prevalencia de osteoporosis y su asociación a factores de riesgo en personas mayores de ambos sexos en Mallorca. Med. Balear. 2007; 22(3): 9-24.

5. Valdivia G, Szot J. Epidemiología de la Osteoporosis. Boletín de la Escuela de Medicina [Internet]. 1999 [citado 2015 Ene 12]; 28(1-2). Disponible en: http://escuela.med.puc.cl/publ/boletin/osteoporosis/EpidemiologiaOsteoporosis. $\underline{\mathrm{html}}$

6. Rosen CJ. Clinical Practice. Postmenopausal osteoporosis. N Eng J Med. 2005; 353(6). 595-603.

7. Organización Mundial de la Salud. Una epidemia intolerable. FHOEMO; 2002.

8. Mendoza S, Noa M, Más R, Fernández L, Illnait J. Manejo de la osteoporosis: enfoque nutricional y farmacológico. Rev. CENIC Ciencias Biológicas. 2005; 36(1):13-22.

9. Guzmán R. Osteoporosis. Nutrición y tejido óseo. Calcio elemental. CES Medicina. 2006; 20(1): 65-75.

10. Izaquirre $Y$, Requeiro J, Machado A, Jiménez $H$, Rodríguez F. La osteoporosis: una epidemia silenciosa. MediSur 2006; 4(1): 85-91.

11. Pérez LI, Alonso A, Roig D, García A, et al. Actualización 2011 del consenso Sociedad Española de Reumatología de osteoporosis. Reumatol Clin. 2011; 7(6): 357-79.

12. Díaz C, Navarro D, Santana F, Domínguez E, Bacallao J. Factores de riesgos modificables o no, relacionados con la densidad mineral ósea en mujeres de edad mediana. Rev. Cubana Endocrinol. 2012; 23(1): 44-55.

13. Maceda WA, Maceda DP, Maceda M, Martinez R, Valcarcel LF. Disminución mineral ósea en pacientes mayores de 50 años y su relación con el IMC. Rev. Diagnóstico [Internet]. 2011 [citado 2014 Dic 25]; 50(3). Disponible en: http://www.fihu-diagnostico.org.pe/revista/numeros/2011/jul-set/123-131.html

14. Calvo IM, Molero J. Factores de riesgo de osteoporosis en mujeres posmenopáusicas. Actualización [Internet]. 2011; 1(3): 121-5.

15. Roig C, Valero D, Romera M, Rozadilla A, Mateo L, Juanola $X$, et al. Prevalencia de los criterios de indicación de densitometría ósea y de los factores de riesgo de baja masa ósea y fractura en unidades extra hospitalarias de reumatología. Rev. Reumatol Clin 2005; 1(1):12-9.

16. López G, Serrano I, Suárez R. Estudio de los factores que predisponen a la osteoporosis en el adulto mayor. Rev. Cubana Reumat. 2012; 14(21): 131-9.

17. Jaller R, Navarro E, Vargas RF. Osteoporosis y factores de riesgo en una población masculina Latinoamericana. Rev. Colomb Reumatol. 2007; 14(2): 99105.

18. González-Pedraza A, Moreno YC, Blanco LA, Medina L, Yánez M, Landgrave $\mathrm{S}$. Factores de riesgo asociados a factores bajos del ultrasonido cuantitativo de calcáneo en adultos mayores en una población del sur de la ciudad de México. Rev. Especial Med. Quirúrg. 2007; 12(3): 30-37.

19. Balderramo DC, Ramacciotti CF, Douthat WG. Factores de riesgo para osteoporosis primaria en mujeres de Córdoba, Argentina. Medicina (Buenos Aires). 2004; 64(5): 400-06. 
20. Padierna JL. Factores de riesgo y prevalencia de osteoporosis estudio por ultrasonometría del calcáneo. Med Int Mex 2008; 24(4): 278-83.

21. De Lago A, Parada MG, Somera J. Prevalencia de osteoporosis en población abierta de la ciudad de México. Ginecol Obstet Mex. 2008; 76(5): 261-6.

22. Bocanegra-Broca YC, Cruz-Rubio FJ, De la Cruz-González C, Jiménez-Sastré A. Prevalencia de osteoporosis y osteopenia en mujeres Tabasqueñas. Salud en Tabasco. 2006; 12(3): 505-13.

23. Ponce $L$, Larenas $G$, Riedemann P. Alta prevalencia de osteoporosis en mujeres mapuches posmenopáusicas asintomáticas. Rev. Médica de Chile 2002; 130(12): 1365-72.

24. Díaz M, García JJ, Carrasca JL, Honorato J, Pérez R, Rapado A, Álvarez C. Prevalencia de osteoporosis determinada por densitometría en la población femenina española. Med Clín. 2001; 116(3): 86-8.

25. Ministerio de Salud. Resolución Nº08430 de 1993. Por medio de la cual se establecen las normas científicas, técnicas y administrativas para la investigación en salud. Bogotá; 1993.

26. Mansini J. Declaración de Helsinki: principios éticos para la investigación médica sobre sujetos humanos. Acta bioethica. 2000: 6(2):321-34.

27. Mosquera MT, Maurel DL, Pavón S. Arregui A, Moreno C, Vázquez J. Incidencia y factores de riesgo de la fractura de fémur proximal por osteoporosis. Rev. panam salud pública 1998; 3(4): 211-9.

28. Padrón RS. Osteoporosis: un problema de salud en aumento. Rev. Cubana Endocrinol [revista en la Internet]. 2001 Ago. [citado 2014 Mar 11]; 12(2): Disponible en: http://scielo.sld.cu/scielo.php?script=sci arttext\&pid=S156129532001000200001\&lng=es.

29. Diez M, Chávez D, Ramírez E, Artega L, Granados R. Estudio de los factores sociomédicos en el Instituto Nacional de Rehabilitación. Rev. mexicana de medicina física y rehabilitación 2009; 19(1):5-7.

30. Du F, Birong D, Changquan H, Hongmei W, Yanling Z, Wen Z, et al. Association of osteoporotic fracture with smoking, alcohol consumption, tea consumption and exercise among Chinese nonagenarians/centenarians. J. Nutr Health Aging. 2011; 15(5): 327-31.

31. Arana E, Gutiérrez I, Ecenarro A, Asua J. Prevalencia de ciertos hábitos determinantes de osteoporosis en mujeres postmenopáusicas del país vasco en 2003. Rev. Esp salud pública. 2007; 81(6): 647-56.

32. González J, Jodar E, Muñoz M, Dies A, Guañabens N. Factores de riesgo de la osteoporosis en mujeres atendidas en Atención Primaria y en consultas hospitalarias. Estudio OPINHO-PC. Rev. Clínica Española. 2009; 209(7): 324-9.

33. Peña A. Efectos del ejercicio sobre la masa ósea y la osteoporosis. Rehabilitacion. 2003; 37(6): 339-53.

34. Glerean M, Plantalech L. Osteoporosis en embarazo y lactancia. Medicina (Buenos Aires). 2000; 60(6): 973-81.

35. Lugones M. Osteoporosis en la menopausia: Prevención y estrategias terapéuticas actuales. Rev. Cubana Obstet Ginecol. 2001; 27(3): 199-204.

36. Barril SM. Afectación articular en la enfermedad inflamatoria articular. Grupo Español de Trabajo en Enfermedad de Crohn y Colitis Ulcerosa (GETECCU). Barcelona; 2002. Disponible en: http://geteccu.org/pacientes/afectacionarticular-en-la-eii 
Recibido: 2 de marzo 2015; Aceptado: 10 de agosto 2015 